\title{
Functional Studies of MLL-AF4 and a Murinized pSer-variant thereof: MLL-AF4 Impairs the Ribosome Biosynthesis Pathway
}

\section{Anna Lena Siemund}

Inst Pharm Biol, Goethe University

Eric Kowarz

Inst Pharm Biol, Goethe University

Rolf Marschalek ( $\square$ Rolf.Marschalek@em.uni-frankfurt.de)

Goethe-Universitat Frankfurt am Main https://orcid.org/0000-0003-4870-3445

\section{Research}

Keywords: MLL-r acute leukemia, MLL, AF4, fusion proteins, pSer domain, SL1, ribosome biogenesis

Posted Date: July 30th, 2021

DOl: https://doi.org/10.21203/rs.3.rs-743930/v1

License: (c) (i) This work is licensed under a Creative Commons Attribution 4.0 International License.

Read Full License 


\section{Abstract}

Background: Recent pathomolecular studies on the MLL-AF4 fusion protein revealed that the murinized version of MLL-AF4, the MLL-Af4 fusion protein, was able to induce leukemia when expressed in murine or human hematopoietic stem/progenitor cells (1). In parallel, a group from Japan demonstrated that the pSer domain of the AF4 protein, as well as the pSer domain of the MLL-AF4 fusion is able to bind the Pol I transcription factor complex SL1 (2).Here, we investigated the human MLL-AF4 and a pSer-murinized version thereof for their functional properties in mammalian cells. Gene expression profiling studies were complemented by intracellular localization studies and functional experiments concerning the biological activities in the nuecleolus.

Results: Based on our results, we have to conclude that MLL-AF4 is predominantly localizing in the nucleolus, thereby interfering withPol I transcription, and subsequently,also ribosomebiogenesis. The murinized pSer-variant is more localizing in the nucleus, which may explain their different biological behavior. Of note, AF4-MLL is cooperating at the molecular level with MLL-AF4, but not with the pSermurinized version of it.

Conclusion: This study provides new insights and a molecular explanation for the known differences between hMLL-hAF4 (not leukemogenic) and hMLL-mAf4 (leukemogenic). While the human pSer domain is able to efficiently recruit the SL1 transcription factor complex, the murine counterpart is not. This has several consequences for our understanding of $t(4 ; 11)$ leukemia which is by far the most frequent leukemia in infants, childhood and adults suffering from $M L L$-racute leukemia.

\section{Introduction}

$M L L-r$ leukemia is diagnosed in $5-10 \%$ of all acute leukemia patients, and the spectrum of $M L L$ fusion partners has increased over the last 30 years of research to more than 100 (3). The most frequent translocation in proB ALL is $\mathrm{t}(4 ; 11)$ (q21;q23) which represents overall about $57 \%$ of all cases. In this particular translocation, the two genes MLL (KMT2A) and AF4 (AFF1) are fused in a balanced recombination event to cause the generation of the two fusion genes MLL-AF4 and AF4-MLL, respectively.

MLL and AF4 wildtype protein complexes have some very basic functions in mammalian cells. The MLL wildtype protein complex is known to confer active chromatin marks on target gene promotors which enables target gene transcription (4-6). The AF4 complex $(7,8)$, also termed "super-elongation complex" (reviewed in ref. 9), is responsible for transcriptional elongation by recruiting and using the P-TEFb kinase $(10-12)$.

In the past decades, researchers have tried to dissect the role of the MLL-AF4 and AF4-MLL fusion proteins. Most studies have failed to demonstrate oncogenic behavior of the MLL-AF4 fusion protein in vitro or in vivo (summarized in ref. 13), except two studies. Lin et al. was able to recapitulate leukemia development in mice when using a partially murinized $\mathrm{h} M L L-m A f 4$ expression construct in distinct target 
cells (1). However, they failed with the full-human counterpart, MLL-AF4, to convincingly create leukemia. Our own study has shown that the AF4-MLL fusion protein is indispensable for leukemia onset, as the onset of leukemia in murine hematopoietic stem/progenitor cells was observed only in the presence of AF4-MLL or both fusion genes, but never with $M L L-A F 4$ alone (14). However, the penetrance was only around $35 \%$ which was partly due to the difficulties in getting efficient packaging of the overlong retroviral vectors constructs, because in the transplantation experiment only 1 in 10,000 hematopoietic stem/precuror cells could be transduced with MLL-AF4 and 1 in 1,000 with AF4-MLL. On the other hand, other transcription factors (e.g. RUNX1) may exhibit complementing functions to substitute the missing MLL-AF4 allele in our experiments (15). Based on our todays knowledge, the AF4-MLL fusion protein exhibits chromatin open functions which may help not only MLL-AF4 to execute the activation of downstream target genes, but also the many other transcription factors that are already expressed in the stem cell compartment $(13,16)$.

It was therefore not surprising that the application of the CRISPR/CAS9 technology to generate balanced chromosomal translocations was sufficient to cause the onset of leukemia in human cord blood cells (17).

In order to understand the role of these fusion proteins, one novel cornerstone was added by the Yokoyama group (2). They unravelled the molecular interaction of the SL1 complex with the pSer domain of AF4 or MLL-AF4. They also dissected the pSer domain at their functional level (18). SL1 represents a transcription factor complex that is composed by $T_{A F} 12, T_{1} F_{1} A, T A F_{\mid} B, T A F_{\mid} C, T A F_{1} D$ and TBP. SL1 is together with UBF - required for Pol I transcription of the ribosomal precursor RNA (45S) that is subsequently processed into $28 \mathrm{~S}, 18 \mathrm{~S}$ and $5.8 \mathrm{~S}$ rRNA (19-23). These rRNA molecules are then used to build up ribosomes to execute protein biosynthesis in cells.

Therefore, we got interested in investigating these novel findings and to find a rational explanation for the inability of human MLL-AF4 to cause leukemia, while the murinized version $M L L-A f 4$ does. To dissect this problem we designed a partially murinized version of human $M L L-A F 4$ by replacing only the human pSer domain with the murine counterpart. This novel construct, $M L L-A F 4 m$, was used along with $M L L-A F 4$ and AF4-MLL to perform all subsequent experiments.

\section{Results}

\section{Construction of transgenes and establishment of stable and inducible cell lines}

We used the already established MLL-AF4 (MA4: MLL ex 1-9::AF4 ex 4-20) and AF4-MLL (A4M: AF4 ex 1-3::MLL ex 11-37) as a starting point to design first the murinized version of MLL-AF4. In order to substitute the corresponding part of the PSER domain, we compared the two homologous sequences that contain the three motifs DLXLS, SDE and NKW (see Fig. 1A). Both sequences are highly homologous, however, the human and murine deviated slightly at certain positions, with 3 missing amino acids in the 
human sequence and 1 missing amino acid in the murine counterpart. Since the latter amino acid was localizing in the SDE motif, we assumed that SL1 binding may be compromized.

By molecular techniques, we substituted the human AF4 sequence coding for AF4 amino acid position $422-555$ by the murine counterpart (422-557) and named this construct MLL-AF4m (MA4m).

Next, we established 5 stable cell lines that express either the 3 fusion gene constructs alone, or in combinations (MA4/A4M and MA4m/A4M). For subsequent experiments, we also constructed a mock control cell line that contained only an empty vector pSBtet-P without Luciferase). After transgene induction, isolated RNA was used to validate correct expression of all 3 transgenes (a 310 bp A4M PCR fragment; a 491 bp MA4 PCR fragment); one primer pair detected specifically the newly integrated murine pSer domain (a 420bp murine pSer PCR fragment; see Fig. 1B). Cells were grown in media to analyze cell viability and growth properties. However, several experiments revealed no significant changes in all 6 established cell lines concerning viability and cell growth (Fig. 1C, left panel). In order to validate functionality of the MA4 and MA4m constructs, a known target gene of MLL-AF4 was tested. As shown in Fig. $1 \mathrm{C}$ (right panel), transcription of HOXA9 could be increased in both cases.

\section{Murinized MLL-AF4 represents a partial loss-of-function variant when binding to SL1}

As described by Okuda et al., 2016, the SDE subdomain of the AF4 pSer doman is responsible for binding the SL1 transcription factor complex. Since both SDE motifs differ slighty between the human and the murine counterpart, we first performed an Co-IP experiment in cells that were stably transfected with either MTM-HA-hum-pSer or the MTM-HA-mur-pSer expression constructs, and transiently transfected with the TAF 1 C::GFP expression construct. Precipitation was carried out with anti-GFP beads, while the detection of precipitated protein was carried out with anti-HA antibodies. As shown in Fig. 1D, the $T_{A F}$ C::GFP protein was able to nearly quantitatively pull-down the MTM-HA-hum-pSer protein, indicative for its strong interaction with SL1. However, $\operatorname{TAF}_{1} \mathrm{C}$ :::GFP could only partially pulled-down with the murinized MTM-HA-mur-pSer protein, indicating for an important difference between both MLL-AF4(m) fusions, namely a near loss-of-function situation with regard to SL1 binding. The difference was about 8fold as shown in the quantification plot (Fig. 1D, right panel).

\section{Murinized MLL-AF4m is unable to synergize with the AF4-MLL fusion protein}

We also performed MACE-Seq studies with all 6 cell lines, and used the mock cell line for normalization of the resulting data. As shortly summarized in Fig. 1E, short term expression of all transgenes (48h) is leading to changes in gene transcription (only $\log 2> \pm 2$ is shown). Co-expression of MA4 and A4M resulted in a synergistic effect with much more genes up-or downregulated when comparing to the single transfected cell line. This synergy effect was completely gone when using the MA4m together with the A4M fusion construct. This indicated for a different mode-of-action of both MLL-AF4(m) fusion protein which needed further investigation. This is also visible from Heatmap (Fig. S1) and Volcano plot analysis (Fig S2). Heatmap analysis shows that MA4 and A4M make synergistic actions and causes strong changes in gene transcription, while the combination of MA4m and A4M did not. Most of signatures on 
co-expressing cells mimics that one of MA4m. Similar results were observed in the volcano plot analysis, where significant MLL (KMT2A) overexpression was visible in A4M expressing cells, while both coexpressing cell lines displayed AF4 (AFF1). Since MACE-Seq is quantitatively amplifying the 3 '-end of RNA,this was an expected result. Noteworthy, AF4 was not visible in the single transfected cell lines expressing MA4 and MAF4, respectivly. This indicated again that the expression of these 2 fusion proteins is much lower without the presence of $A 4 M$.

\section{MA4 localizes to the nucleolus, MA4m to nucleus and nucleolus, but only MA4 redirects RNA Pol II from the nucleus to the nucleolus}

Since both constructs, MA4 and MA4m, were able to interact with SL1 we got interested into the subcellular localization of both fusion proteins. Therefore, we cloned an in-frame mCherry-Tag to the Cterminal portions of both MA4 and MA4m vector constructs. Both constructs were again stably transfected and transgene expression was induced by adding $1 \mu \mathrm{g} / \mathrm{ml}$ Doxycyclin to analyze their intracellular localization. As shown in Fig. 1F, mock-transfected cells were stained by an antibody against the important UBF factor which is necessary for RNA Pol I transcription. As shown in these experiments, UBF localizes precisely to the nucleoli of the investigated cells. Next, we analyzed the distribution of MA4::mCh and MA4m::mCh fusion proteins. Surprisingly, MA4 localizes solely in nucleoli, while MA4m displayed besides the nucleolar localization also a staining of the nucleus. In addition we investigated also their capacity to bind to Pol I promotor structures, however, we could not see any binding of both fusion proteins to these promotor structures when analyzing the mCherry-pulled-down genomic DNA by Q-PCR experiments (Fig. F, right panel). Next we investigated the localization of RNA Pol II distribution. As shown in Fig. 1G, RNA Pol II is distributed all over the nucleus in mock-transfected cells, however, this picture changed dramatically, when analyzing MA4- or MA4m-transfected cells. In MA4-transfected cells, RNA Pol II localizes predominantly to the nucleolus, while RNA Pol II localizes in part to the nucleolus, but mainly in the nucleus in MA4m-transfected cells. This was also quantified experimentally, by analyzing the Pol II-pulled-down genomic DNA in Q-PCR experiments for the presence of rRNA gene promoter sequences. Of interest, we were able to demonstrate that RNA Pol II was bound to the Pol I promoters in MA4-transfected cells (Fig. 1G, right panel), while this was not the case when analyzing the MA4mtransfected cells. From these data we concluded that MA4 is mainly localizing within the nucleolus, and seems to be responsible for the relocalization of RNA Pol II from the nucleus to the nucleolus, resulting in bound RNA Pol II at rRNA promoter sequences which are usually bound by RNA Pol I. Therefore, a competing situation is being created between RNA Pol I and RNA Pol II.

\section{Changes in the morphology of nucleoli and rRNA promoter activity}

High resolution microscopy was used to study the structure and amount of nucleoli in the MA4- and MA4m-transfected cell lines. As shown in Fig. 2A, the morphology of nucleoli is changing when either of these both fusion proteins were expressed. The relative protein level of UBF did not change significantly when nucleoli were stained by an anti-UBF antibody and quantitatively analyzing the fluorescence signals of the secondary antibody. However, the number of nucleoli became reduced - even significantly - when 
MA4 is expressed (see Fig. 2A, right panels). The highly condensed structure became larger and was smoothened. Therefore, we asked the question whether RNA Pol I mediated transcription of the 45S precursor becomes affected. For this purpose, a Pol I-dependend Luciferase reporter system was established (see Fig. 2B). This reporter system is based on a single rRNA gene promotor fragment (744bp) that was cloned in front of an IRES and a firefly Luciferase reporter gene. A construct without the RNA Pol I promotor fragment was uses as negative control, and an SV40 promoter with Renilla Luciferase served as internal normalization vector for our transfection experiments. The reporter system was functionally tested in a proof-of-principle experiment, namely by adding 2,5 nM - $10 \mathrm{nM}$ Actinomycin D (ActD), a known inhibitor of RNA Pol I transcription (see Fig. 2B, lower right panel). Vice versa, overexpression of a transiently transfected $\mathrm{TAF}_{\mid} \mathrm{C}::$ GFP led to an increase of rRNA promoter activity (see Fig. 2C, left panel). Next, transient transfection experiments with the rRNA promoter construct \pm the $\mathrm{TAF}_{\mid} \mathrm{C}::$ GFP expression construct into the 6 different cell lines revealed a first important result: MA4 - and to a lesser extend MA4m - caused a significant reduction of rRNA gene transcription that could be reversed by the presence of $\mathrm{TAF}_{1} \mathrm{C}: . \mathrm{GFP}$ (see Fig. $2 \mathrm{C}$, right panel). This phenomenon was even enhanced further in the presence of co-expressed $A 4 M$ which caused a reduction to $55 \%$. Thus again, the import of RNA Pol II from the nucleus into the nucleolus seems to impair proper RNA pol I activity, if not compensated by additional $\mathrm{TAF}_{\mid} \mathrm{C}$.

In order to validate these unexpected results, we also investigated the relative expression of 45S rRNA by Q-PCR (see Fig. 2D, left panel). Surprisingly, although the rRNA promoter activity was found to be reduced when using our reporter construct, the steady-state amount of 45S rRNA was actually increased. This controversial results could be explained either by quenching effect (rRNA promoters vs. reporter plasmids), or, by a more frequent use of very specific of endogenous rRNA promoter sequences $(n=400)$, which are not all identical. However, the protein level of UBF was unchanged when nucleoli were stained by an anti-UBF antibody and quantitatively analyzing by the fluorescence signals of the secondary antibody (see Fig. 2D, right panel).This indicated that the actual number of rRNA gene copies used for gene transcription remained unchanged. Usually, cells use only about half of their 400 rRNA gene copies for gene transcription by RNA Pol I (24). Thus, we remained at this point with an enigmatic experimental result.

\section{Influence Of Ma4 And Ma4m On Protein Biosynthesis}

Having more 45S rRNA being produced, we were interested in finding out the effect on protein biosynthesis. By using the commercially available Click-it protein synthesis assay (Thermo Fischer Scientific), we were able to demonstrate that stably transfected cells expressing MA4 - but even more when expressing MA4m - the protein biosynthesis seems to be impaired (see Fig. 2E). Co-expression of MA4 and A4M had the strongest effect, while the combination of MA4m and A4M seem to cause less inhibition of protein biosynthesis. In all these cases, the negative effects of the single fusion proteins - or when co-expressed with $A 4 \mathrm{M}$ - on protein biosynthesis were reversed by additional expression of transient $\mathrm{TAF}_{\mid} \mathrm{C}:: \mathrm{GFP}$. 


\section{Nucleolar stress caused by MA4 and MA4m blocks apoptosis and causes the activation of the WNT/ß- catenin pathway}

In order to understand the molecular consequences in more detail, we investigated the role of nucleolar stress conditions when both fusion proteins, MA4 or MA4m, were expressed in mammalian cells (25). In Fig. $2 \mathrm{~F}$, the nucleolar stress pathway is shown on the left. If cells are unable to produce enough 45S RNA $(28 \mathrm{~S}, 18 \mathrm{~S}, 5.5 \mathrm{~S})$, supernumerary ribosomal proteins not being involved in ribosome subunit production are able to bind nuclear MDM2, which usually binds and triggers the proteasomal degradation of p53. Thus, the nucleolar stress response leads to an accumulation of p53, which in turn causes usually a p21dependent cell cycle arrest and apoptosis. In addition, inhibited production of 45S RNA - or its subseqent processing into $28 \mathrm{~S}, 18 \mathrm{~S}$ and $58 \mathrm{~S}$ rRNA (by PPAN, NPM, PES1 or SBDS) - is leading to an accumulation of cytosolic ß-Catenin, which in turn causes the upregulation of pro-survival WNT/ß-Catenin target genes to bypass this stress conditions (26).

We could demonstrate experimentally that the steady-state protein accumulation of p53 and ß-Catenin when mock cells were treated with 2,5-10 nM ActD (positive control, see Fig. 2F, middle upper and lower left panel). When analyzing the 6 cell line, p53 was strongest induced either by MA4m alone, or by the combination of MA4 and A4M. Of interest, this stress phenomenon was gone when MA4 was coexpressed with $A 4 \mathrm{Mm}$ (see Fig. 2F, upper right panel). In addition, an increase of $B$-Catenin was observed strongest with MA4m alone or by the combination of MA4 with A4M (see Fig. 2F, lower middle panel). A common pro-survival WNT/ß-Catenin target gene of this nucleolar stress pathway is HOXB4, which was induced in all 5 cell lines expressing $t(4 ; 11)$ fusion proteins, indicating that the presence of these fusion proteins, including the artificial MA4m fusion construct, are all triggering this nucleolar stress response pathway, presumably to prevent p53-mediated apoptosis.

\section{Discussion}

This paper aimed to get new insights into the differences between the human and murine AF4 protein portion which - when fused to the N-terminal portion of MLL - may allow the development of leukemia (1). A first hint came from studies on AF4 and SL1, a transcription factor complex that is necessarily involved in the transcription of ribosomal RNA in mammalian cells (19-23). SL1 is composed by TAF, $12, T_{A} F_{\mid} A$, $T A F_{\mid} B, T A F_{\mid} C, T A F_{\mid} D$ and $T B P$. Of note, $T A F_{\mid} C$ has already been shown to exhibit the strongest binding to the PSER domain which made it attractive to use it as experimental read-out system (2). These studies, which dissected the process of SL1 binding to specific subdomains of the AF4 pSer domain, brought some new ideas to the enigmatic stories about the MLL-AF4 fusion protein that derived from the balanced chromosomal translocation $\mathrm{t}(4 ; 11)(18,27)$. The pSer domain of AF4 is always fused to the Nterminal portion of MLL in all yet investigated patients with a $t(4 ; 11)$ translocations, regardless of the breakpoint localization within the $M L L$ gene (3). It contains the three subdomains DLXLS, SDE and NKW, respectively. SDE and NKW were shown to be important for transactivation and transforming ability, with SDE as SL1-binding platform and NKW needed to initiate RNA Pol II-dependent transcription. 
Since Lin et al. used the complete Af4 sequence in their hMLL::mAf4 construct to express the MLL-Af4 fusion protein, we decided to investigate SL1 binding to AF4/Af4 sequences as a potential explanation for their observed differences in leukemia onset and development (1).

Our first attempt to compare the pSer domain between both orthologous protein sequences already identified some minor differences which could be tested in our planned experimental setting. As shown in Fig. $1 \mathrm{~A}$, the SDE subdomain known to recruit the SL1 complex exhibits a missing single amino acid in the murine sequence. Therefore, we substituted a portion of the AF4 pSer domain (134 amino acids) within the MLL-AF4 (MA4) by the orthologous mouse sequences (136 amino acids). This novel construct was termed MLL-AF4murine (MA4m). Having done this, we used our established Sleeping Beauty technology (28) to generate a series of stably transfected cell lines that allows to investigate potential differences in MA4- and MA4m-transfected cell lines.

The first important finding was the binding of the SL1 complex to both pSer domains. We used not the full-length MA4(m) constructs, but a mini-MLL-AF4 version designed in Akihiko Yokoyama's lab (2). By

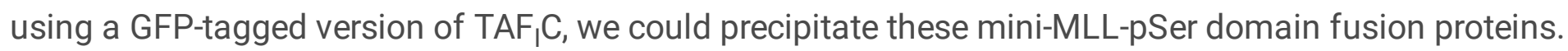
However, while the human mini-MLLpSer was strongly precipitated, the murine counterpart did not effectively bind to $T_{A F} C$ (see Fig. 1D). The quantification revealed that murine pSER binds 8-times less efficient than the human pSer domain. Both domains can be distinguished by a single missing amino acid (SDE: SES-(T)-SDSDSSSDSE).

When we analyzed the intracellular localization of both full-length fusion proteins, MA4 and MA4m, we had another surprise: although we know that MLL-AF4 fusion proteins are distributed in the cell nuclei to bind to their specific target genes, the majority of the fusion protein was localizing in the nucleolus (Fig. 1F). This was true for MA4, and to a lesser extent also for MA4m. This may indicate for a competitive situation of both fusion proteins, namely to bind to nuclear target genes, and in addition, to enter the nucleolus where they both have a yet unknown function. Noteworthy, more available MA4m in the nucleus will allow a more effective binding to target genes which could be an explanation for the finding of Lin et al. (1). Of interest, only in the presence of MA4, RNA polymerase II could be precipitated on rRNA promoters (Fig. 1G, right panel). Thus, in the presence of MA4 - but not with MA4m - a large amount of RNA Pol II seems to be redirected from the nucleus to the nucleolus (Fig. 1G, left panels).

This has been investigated as well by a straight forward MACE-Seq experiment, where we only wanted to see the differences in a genome-wide fashion. Based on the 6 established cell lines (see Fig. 1B), we used biological triplicates to investigate the target gene spectrum of MA4 alone, MA4m alone, A4M alone and the 2 co-expressing cell lines. In the VENN diagram shown in Fig. 1E, the upregulated target genes in MA4, A4M and MA4/A4M clearly pointed to a synergistic activity of both reciprocal fusion proteins. The idiosyncratic target gene spectrum with MA4m was strongly increased (from 34 to 84), but the synergistic cooperation with A4M was lost. Similar observations have been made for the downregulated target genes. 
Therefore, we looked to the nucleoli in more detail to just realize that their compact and condensed structure, known to many scientist, becomes less defined and more smoothened in the presence of both MA4 and MA4m (see Fig. 2A). Despite these changes in morphology, also the number of nucleoli becomes reduced, while the amount of the UBF protein, binding to all active rRNA gene promoters remained constant in all investigated cells (Fig. 2A, right panel).

In order to set up novel tools to investigate rRNA promoter activity in a quantitative fashion, we designed a novel reporter system. One of the many repetitive rRNA promoters was cloned that contained the binding sites for UBF (upstream element UE and core element) and SL1 (core element CE) as well as the transcriptional start site (TSS; see Fig. 2B). This promoter element was cloned into a Luciferase reporter vector that contains an IRES sequence which allowed us to measure Luciferase activity caused by RNA Pol I transcription. By using this reporter construct (together with a negative and internal control), we could show that the addition of up to $10 \mathrm{nM}$ Actinomycin D - a poison for RNA Pol I transcription functionally impaired RNA Pol I transcription. By contrast, the transient overexpression of a TAF, C::GFP fusion protein increased the relative rRNA promoter activity (see Fig. 2C). Subsequently performed experiments with all 6 stable cells lines revealed that MA4 and both co-expressing cell lines displayed a much lower Luciferase activity. MA4m did show the inhibitory effect to a lesser extent, but the transient co-expression of the TAF, C::GFP construct reverted the observed inhibitory effects. This may indicate that binding of SL1 to MA4 causes an out-competition of SL1, so less is available for rRNA production by RNA Pol I and the reporter gene plasmid, while MA4m has less power to attract SL1 away from its natural promoter (as already shown in Fig. 1D), or the additional reporter gene construct. In addition, the presence of A4M made things even worse, as here only 50\% reporter activity could be reached when compared to mock cells. How A4M is causing this augmented inhibitory activity is currently unknown.

Any reduction of rRNA synthesis has dramatic effects on cells. Although 95\% of all RNA produced in mammalian cells is the 45S rRNA precursor which is then processed into the $28 \mathrm{~S}, 18 \mathrm{~S}$ and $5.8 \mathrm{~S}$ rRNA molecules. These rRNAs are then used to build up ribosomes inside of the nucleoli, and to transport the $60 \mathrm{~S}$ and $40 \mathrm{~S}$ ribosomal subunits across the nucleus to a nuclear pore where both subunits become exported to the cytosol for protein biosynthesis. Cells do not tolerate changes to this important ribosome biosynthesis system and are very sensible to even tiny changes.

Therefore, we investigated cytosolic protein biosynthesis in all 6 cell lines (see Fig. 2E). The assay revealed a quite similar picture as in Fig. $2 \mathrm{C}$, because protein biosynthesis was impaired in these cells. Again, all inhibitory effects could be complemented by the transient overexpression of the TAF,C::GFP fusion protein.

To analyze the nucleolar stress pathway in more detail, we also investigated the steady-state abundance of p53 and B-Catenin. As outlined in Fig. 2F, nucleolar stress is translated into the stabilization of p53 leading to cell cycle arrest and apoptosis. Recently, a novel nucleolar stress mechanism could be identified that causes the stabilization of cytosolic ß-Catenin, which then allow to bypass the adverse effects of p53 (cell cycle arrest and apoptosis). By contrast, the ß-Catenin signaling activity leads to the 
transcriptional activation of prosurvival genes like HOXB4 (26). Therefore, we analyzed the protein levels of p53 and B-Catenin in Western Blot experiments, as well as the expression of HOXB4 by Q-PCR. The results of these experiments indicated that all $t(4 ; 11)$ fusion proteins - or variants thereof - result in nucleolar stress reaction and downstream target gene activation (exemplarily shown for HOXB4).

Thus, we have to conclude that fusion proteins deriving from $t(4 ; 11)$ translocations are able to activate specific target genes in the nucleus (MA4, MA4m), change chromatin accessibility (A4M), or show cooperative effects when co-expressed (13). In addition, these fusion proteins seem to impair the Pol I transcriptional pathway and inflict with normal protein biosynthesis in mammalian cells. This makes these fusion proteins quite toxic, which could also explain why MLL-AF4 is always very low-abundantly expressed in mammalian cells, and enhanced expression is even leading to a cell-cycle arrest (29).

Probably $t(4 ; 11)$ cells may use the nucleolar stress pathway to enhance $ß-C a t e n i n$ signaling. The WNT/ßCatenin signaling pathway has already been identified as key pathway in MLL-r acute leukemias. GSK3ß and $B$-Catenin are necessary for $M L L$-r leukemia cells allow formation of leukemic stem cells and to induce additional survival pathways (30-32).

The only result we could not explain is shown in Fig. 2D. While all our results point to a deregulation of nucleolar activity and even perturbation of protein biosynthesis, this result showed an increased production of $45 S$ RNA. Whether this is a compensatory mechanism of cells expressing $t(4 ; 11)$ fusion proteins, e.g. by using an increasing amount of the 400 rRNA transcription units, remained enigmatic. However, an interesting observation has been made recently when studying ribosome biosynthesis (33). RNA Pol II is quite important in nucleoli for the biosynthesis of rRNA, because RNA Pol II - associated with Senataxin (SETX) - transcribes intergenic regions between rRNA genes, and thus, inhibits the formation of sense intergenic noncoding RNA (sincRNA) produced by RNA Pol I. This kind of "shielding effect" is caused by R-loop formation during transcription of RNA Pol II which in turn allows to maintain high yields in rRNA production. Thus, RNA Pol II inside of nucleoli has a quite important biologcal function. MA4 seems to enhance this relocalization process, however, is presumably disturbing the shielding effects of natural RNA Pol II, because we observed RNA Pol II at RNA Pol I promoters, a drop of rRNA promoter activity and decreased protein biosynthesis.

\section{Conclusions}

Several questions are still remaining, however, this manuscript shows for the first time that $t(4 ; 11)$ fusion proteins - or variants thereof - are influencing one of the most sensible pathways in our cells, namely ribosome biogenesis. Future work has to be carried out to deepen these insights in order to understand the impact of these fusion protein, not only in the nucleus, but also in the nucleolus of affected cells.

Taken together, we identified that the t(4;1)-derived MLL-AF4 fusion shows (1) cooperativity with the reciprocal AF4-MLL fusion protein, (2) recruits and binds strongly to the SL1 transcription factor complex, (3) targets and deregulates nuclear genes, (4) however, is mainly localized in the nucleolus, (5) recruits RNA Pol II into the nucleolus and (6) interfers with ribosome biogenesis. Exchanging the pSer domain 
within the MLL-AF4 fusion protein by the murine homologous sequences ( -130 amino acids) nearly (7) abolishes the recruitment of SL1 to MLL-AF4m and (8) localizes now more in the nucleus of cells where this partly murinized fusion protein (9) deregulates more target genes. These data explain the differences in the leukemogenic behavior of MLL-AF4 (not leukemogenic) and hMLL-mAF4 (which is leukemogenic) at the molecular level. Moreover, this study provide insights into a completely new pathway important for cancer cells: interfering with ribosome biosynthesis is leading to the activation of the WNT/ß-Catenin pathway which is necessary for leukemic stem cells formation.

\section{Methods And Materials}

\section{Cell culture and transfections}

HEK293T cells were grown in DMEM with 10\% (v/v) FCS (Capricon Scientific), 2 mM L-Glutamine (Capricon Scientific), and $1 \%\left(\mathrm{v} / \mathrm{v}\right.$ ) Pen Strep (GE Healthcare) at $37^{\circ} \mathrm{C}$ and $5 \% \mathrm{CO}_{2}$. Stable cell lines were established using an optimized Sleeping Beauty Transposon System (28). $50 \mathrm{ng}$ of SB transposase vector SB100X and $1 \mu \mathrm{g}$ of the respective plasmid(s) were applied with Metafectene Proß (Biontex) (pSBtet::MLL-AF4, pSBtet::MLL-AF4m, pSBtet::AF4-MLL, pSBtet::TAF,C::GFP). After 24 hours, cells were subjected to either Puromycin (AF4-MLL, $2 \mu \mathrm{g} / \mathrm{ml}$ ) or Blasticidine (MLL-AF4, MLL-AF4m; $15 \mu \mathrm{g} / \mathrm{ml}$ ). The cells were incubated with selection markers for 3-10 days. Transgene induction was carried for at least $48 \mathrm{~h}$ with $1 \mu \mathrm{g} / \mathrm{ml}$ Doxycycline. Another cell line was created by stable transfection of the pSBtet-P vector (without Luciferase) and used throughout the experiments as mock control.

\section{Plasmid Constructions}

The plasmid encoding the MTM-HA-hum-pSer gene was kindly provided by Akihiko Yokoyama (Tokyo, Japan; 2), and was used to replace to human pSer domain by the murine counterpart to obtain the MTMHA-mur-pSer construct. Both constructs were cloned via Sfi1 sites into pSBtet-P.

The $T A F_{1} C::$ GFP construct was designed by fusing the open reading frames of $T A F_{1} C$ with that one of super-folder GFP (pET29BH4:10xHis-TEV-sGFP was a gift from Dr. Jan Hering, Frankfurt, Germany) to obtain the final constructs. This construct was cloned via $S f 11$ sites into pSBtet-B. TAF $C$ is central part of SL1 and was already shown to bind strongest to the pSer domain of AF4 (1), and thus, was used in all experiments to represent SL1 binding to MA4 or MA4m.

The MA4::mCh and MA4m::mCh constructs were designed by eliminating the terminal stop codon and fusing the open reading frame to the mCherry open reading frame. The two final constructs were cloned into pSBtet-P.

The rRNA promoter sequence was cloned by PCR from the human genome with the 2 oligonucleotides pHrRNA.F (5'-CACCTCGAGCGCGATCCTTTCTGGAGAGTCCC-3') and pHrRNA.R (5'-

AAGCGAATTCGACGAGAACGCCTGACACGCAC-3'), digested with Xhol and EcoRI and cloned as a 754 bp 
long DNA fragment into the pGL3-IRES-Basic (Addgene) to obtain a Pol I Luciferase reporter plasmid. pGL3-IRES served as negative control. A SV40-Renilla Luciferase construct served as internal standard for all experiments. The ribosomal promoter element contained the upstream element (binding site for UBF) and the core element (binding site for UBF and SL1), as well as the transcriptional start site.

\section{Rna Extraction, Cdna Synthesis And Rt-pcr Experiments}

RNA extraction, cDNA synthesis and RT-PCR experiments In all 6 stable cell lines, transgene induction was carried by using $1 \mu \mathrm{g} / \mathrm{ml}$ Doxycycline for 48 hours. Total RNA was isolated by using RNeasy ${ }^{\circledR}$ Mini Kit (Qiagen) and cDNA synthesis were performed using SuperScript ${ }^{\circledR}$ II (Invitrogen). All isolated RNAs were quality checked (Agilent Bioanalyzer) and final concentrations were determined. Equal amounts of total RNA were used throughout all experiments, and all experiments were performed with 3 biological replicates. Primers used for RT-PCR analyses are as follows: A4M.F 5'-TCCGGCCCATGGATGGTCAAGATCAGGC-3', A4M.R 5'-

TTGTGGAAGGGCTCACAACAGACTTGGC-3', MA4.F 5'-ACCTACCCCATCAGCAAGAGAGGATCCTGC-3', MA4.R 5'-GCCATGAATGGGTCATTTCCTTCAGAATCT-3', Af4.pSer.F 5'-

CGTCTCCATGCTGGAGGACGACCTGCAGCTCAG-3' and Af4.pSer.R 5'AGAATGCTCCTGGTCACTGCTGCCCTCAGCGACA-3'. Target gene transcription was quantified in Q-PCR experiments with the following primers: HOXA9.F (5'-CAATGCTGAGAATGAGAGCGG-3'), HOXA9.R (5'TGTATAGGGGCACCGCTTTTT-3'); HOXB4_RT.F (5'-CCTGGATGCGCAAAGTTCAC-3'), HOXB4_RT.R (5'CCTTCTCCAGCTCCAAGACC-3'), GAPDH.F 5'-GGTCACCAGGGCTGCTTTTA-3', GAPDH.R 5'CGTTCTCAGCCTTGACGGTG-3', qPCR_45SrRNA_28S.F (5'-CGATCTATTGAAAGTCAGCCCTCGACACAAGG3') and qPCR_45SrRNA_3'ETS.R (5'-CGGTCGGCGGGAGAGGCCGGGAGGGAGGAAGACGAACG-3').

\section{Differential Gene Expression Profiling By Mace-seq}

For the MACE-Seq experiments, all cell lines were treated with $1 \mu \mathrm{g} / \mathrm{ml}$ Doxycycline for $48 \mathrm{~h}$ with and total RNA were isolated from transfected cell lines. After testing the correct expression of transgenes, differential gene expression (DGE) profiles were obtained by MACE (Massive Analysis of cDNA Ends) Seq experiments following the manufacturer protocol (GenXPro, Frankfurt, Germany). Three biological replicates of each cell line were compared with 3 biological replicates of mock-transfected cells. The MACE-libraries were prepared at GenXPro GmbH using the Massive Analysis of cDNA Ends (MACE) Library Preparation Kit (v2.0) from GenXPro GmbH. First, cDNA was generated using Oligo(dT) primers with distinct Oligo IDs per sample for subsequent pooling of up to 24 samples. After pooling, cDNA was fragmentated to an average size of $200 \mathrm{bp}$ using the sonicator Biorupter Plus (Diagenode, Belgium). The distribution of CDNA fragment sizes was monitored using the automated microfluidic electrophoresis station LabChip GXII Touch HT platform (PerkinElmer, USA). The Poly(A) containing cDNA fragments were purified using solid phase reversible immobilization (SPRI) beads (Agencourt AMPure XP, USA), end repaired and ligated to distinct 8-base pair UMI Adapters (also called TrueQuant adapters). Then, the 
library containing labelled and fragmentated cDNA was amplified by PCR, purified by SPRI beads (Agencourt AMPure XP, USA) and strand-specific sequenced using the HiSeq2500 (Illumina, USA).

Bioinformatic analysis was performed according to the analysis pipeline for MACE libraries by GenXPro $\mathrm{GmbH}$. Unique Oligo IDs and UMIs on each transcript allowed initial demultiplexing and subsequent removal of PCR-duplicates. The remaining reads were trimmed for high-quality as well as adapter-free sequences and aligned to the human reference genome (Genome Reference Consortium Human Build 38 patch release 13 (GRCh38.p13) using Bowtie 2. Resulting output data were implemented in the database program FileMaker for further analysis. All data received from the Bioconductor software from the MACESeq experiments were incorporated into a FILEMAKER database program. In addition, we used the following server for further data analysis: Heatmapper (http://www.heatmapper.ca/expression/) for heatmap analyses and VolcaNoseR (https://huygens.science.uva.nl/VolcaNoseR/) for volcano plots.

\section{Antibodies Used Throughout This Study}

The following antibodies have been used throughout this study: anti- $\beta$-Catenin (Cell Signaling, \#8480), anti HA-Peroxidase (Sigma Aldrich, \#34071100), anti rabbit IgG-Peroxidase (Abcam, ab6721; secondary antibody, Western Blot), anti mouse IgG-Peroxidase (Abcam, ab97023; secondary antibody, Western Blot), anti GFP(Abcam, ab290), anti UBF (Santa Cruz, sc-13125), anti mCherry (Abcam, ab125096), anti $\beta$-ActinPeroxidase (Sigma Aldrich, A3854), anti RNA Polymerase II (Diagenode, AC-055-100), anti mouse IgGAlexa Fluor®586 (Abcam, ab175473; secondary antibody, IHC), anti p53 (Santa Cruz, sc-47698), respectively.

\section{Cell Fixation And Immunofluorescence Staining And Detection}

HEK 293T cells lines were cultivated on Poly-D-Lysin pretreated glass chamber slides and transgene expression was induced for $48 \mathrm{~h}$ with $1 \mu \mathrm{g} / \mathrm{ml}$ Doxycycline. Next, cells were washed with PBS containing 1 $\mathrm{mM} \mathrm{CaCl}_{2}$ and $0,5 \mathrm{mM} \mathrm{MgCl}_{2}$ and then fixed for $20 \mathrm{~min}$ in cell fixing solution (3,7\% Formaldehyde (v/v) in PBS + $1 \mathrm{mM} \mathrm{CaCl}_{2}, 0,5 \mathrm{mM} \mathrm{MgCl}_{2}$ ) following quenching in $50 \mathrm{mM} \mathrm{Glycin} \mathrm{in} \mathrm{PBS}+1 \mathrm{mM} \mathrm{CaCl}_{2}, 0,5 \mathrm{mM}$ $\mathrm{MgCl}_{2}$ for $5 \mathrm{~min}$. After repeated washing with PBS, cells were permeabilised for $15 \mathrm{~min}$ in a permeabilisation solution (0,2 \% Triton $^{\mathrm{TM}} \mathrm{X}-100,0,1 \% \mathrm{SDS}$ in PBS $\left.+1 \mathrm{mM} \mathrm{CaCl}_{2}, 0,5 \mathrm{mM} \mathrm{MgCl}_{2}\right)$.

In case of immunostaining, glass slides with fixed cells on the surface were blocked in a Coplin Jar with TBST with $5 \%$ BSA for $1 \mathrm{~h}$ and afterwards incubated in TBST diluted primary antibody o/n at $4^{\circ} \mathrm{C}$. The next day cells were washed with TBST and incubated in TBST diluted secondary antibody for $1 \mathrm{~h}$ at RT. After repeated washing with TBST stained cells were embedded in Duolink $\circledast$ In Situ Mounting Medium with DAPI (Sigma Aldrich) and analysed with the fluorescence microscope Observer Z1 (Carl Zeiss). 
For Quantification of UBF protein levels $1 \times 10^{4}$ cells of each stable transfected HEK 293T cell line were seeded in triplicates in a 96-well plate and incubated for $48 \mathrm{~h}$ with Doxycycline. After the fixation procedure as mentioned above, an antibody incubation was carried out in TBST o/ $\mathrm{h}$ and for $1 \mathrm{~h}$ respectively. After washing with TBST, $50 \mu \mathrm{L}$ of HCS NuclearMask ${ }^{\mathrm{TM}}$ Blue Stain $(\mathrm{H} 10325$, Thermo Fisher Scientific) was added per well and incubated $30 \mathrm{~min}$ at RT protected from light. Afterwards, all wells were washed again and $100 \mu \mathrm{l}$ of TBST was added before measuring flourescense signals at the Varioskan Flash plate reader (Thermo Fisher Scientific). The analysis was performed by normalization to DAPI and mock.

\section{Q-pcr Experiments}

All quantitative PCR analyses were performed with the StepOnePlus ${ }^{\text {TM }}$ System (Applied Biosystems). All measurements were normalized to the $\mathrm{Ct}$ values of GAPDH of mock transfected cells and were analyzed in triplicates. The results were evaluated by the comparative $\Delta \Delta \mathrm{Ct}$ method.

\section{Viabilitätsassay}

For the determination of cell viability $1 \times 10^{6} \mathrm{HEK} 293 \mathrm{~T}$ cells were seeded into $10 \mathrm{~cm}$ cell culture dishes and transgene expression was induced for $48 \mathrm{~h}$ with $1 \mu \mathrm{g} / \mathrm{ml}$ Doxycycline. Cells were detached by Accutase ${ }^{\circledR}$ (Capricorn) treatment and an Aliquot was mixed with Acridine Orange and DAPI containing Solution (Chemometec) and analysed with the Nucleocounter $\mathrm{NC} 3000^{\mathrm{TM}}$ (Chemometec) according to manufactors instructions.

\section{Luciferase Reporter Assay}

The rRNA promoter acitvity was measured using the Dual-Luciferase ${ }^{\circledR}$ Reporter Assay System" from Promega. $4 \times 10^{5}$ HEK 293 T cells were seeded in a 6-well plate in triplicates. The expression of transgenes was induced by the addition of $1 \mu \mathrm{g} / \mathrm{ml}$ Doxycycline for $48 \mathrm{~h}$. $24 \mathrm{~h}$ prior to analysis, cells were transiently transfected with reporter and control vectors. Measurement of Luciferase activities was performed according to manufactors instructions.

\section{Western Blot}

$5 \times 10^{5}$ cells of each HEK $293 \mathrm{~T}$ cell line were cultivated in 6 -well plates for $48 \mathrm{~h}$ with $1 \mu \mathrm{g} / \mathrm{ml}$ Doxycycline for induction of transgene expression. Afterwards cells were lysed for $45 \mathrm{~min}$ in $50 \mu \mathrm{l}$ lysis buffer ( $1 \%$ Triton X-100 (v/v), 1\% Deoxycholat (w/v), 1 x protease inhibitor cocktail (Roche) at $4^{\circ} \mathrm{C}$. Cell lysates were obtained after centrifugation at $13.000 \mathrm{rpm}$ for $10 \mathrm{~min}$. Whole cell lysate was loaded onto a $10 \% \mathrm{SDS}$ Gel. Seperated proteins were transferred onto a PVDF membrane using the standard protocol for Trans- 
Blot TURBO system (BioRad). After blocking in TBST + $5 \%$ BSA for $1 \mathrm{~h}$ at RT, membranes were incubated in primary antibody $\mathrm{o} / \mathrm{n}$ at $4^{\circ} \mathrm{C}$. The next day membranes were washed in TBST and incubated in secondary antibody for $1 \mathrm{~h}$ at RT following detection using the Clarity ${ }^{\mathrm{TM}}$ ECL Western substrate and Chemi DOC ${ }^{\mathrm{TM}} \mathrm{XRS}+$ Imager (Biorad).

\section{Co Immunoprecipitation}

$1 \times 10^{7}$ HEK 293T cells were seeded into a $15 \mathrm{~cm}$ cell culture dish with $1 \mu \mathrm{g} / \mathrm{ml}$ Doxycycline for induction of transgene expression for $48 \mathrm{~h}$. The Medium was discarded, cells were washed with ice cold PBS, resuspended in lysis buffer (150 mM NaCl, 10 mM Tris-HCl, 1 mM EDTA, 1 mM EGTA, 1 \% Triton X-100 (v/v), 0,5\% NP40 (v/v), 1x Protease Inhibitor Cocktail (Roche)) and incubated for 30 min on ice. After centrifugation at $1000 \mathrm{rpm}$ for $5 \mathrm{~min}$ at $4^{\circ} \mathrm{C}$ the cell lysate was transferred into a new $1,5 \mathrm{ml}$ reaction tube. Protein concentration was determined with Pierce ${ }^{\mathrm{TM}}$ BCA Protein Assay (Thermo Fisher Scietific) and 200 $\mu \mathrm{g}$ protein was used per IP which was performed according to NEB protocol.

\section{Chromatin Immunoprecipitation Experiments}

ChIP experiments were performed using the Abcam protocol. Stably transfected HEK 293T cells $\left(1 \times 10^{7}\right.$ cells on a 145-mm cell culture plate) were transgene-induced with $1 \mu \mathrm{g} / \mathrm{ml}$ Doxycycline for $48 \mathrm{~h}$. For double fixation, the cells were incubated with $2 \mathrm{mM}$ di(N-succinimidyl)glutarate for 45 minutes and $1 \%$ $(\mathrm{v} / \mathrm{v})$ formaldehyde for 10 minutes. Sheared chromatin was incubated with magnetic $A / G$ beads and antibodies overnight following precipitation. Quantitative PCR analysis was performed with the percent input method from ThermoFisher Scientific by using the following primers: rRNA.Prom.for (5'GGCTGCGATGGTGGCGTTTTTGG-3') and rRNA.Prom.rev (5'-GGACAGCGTGTCAGCAATAACCCG-3').

\section{Click It Protein Synthesis Assay}

The analysis of protein biosynthesis in HEK 293T cellines was performed with $2 \times 10^{4}$ cells in each PolyD-Lysin pretreated 96-well plates after $48 \mathrm{~h}$ induction with $1 \mu \mathrm{g} / \mathrm{ml}$ Docxcyclin with the "Click-iT ${ }^{\mathrm{TM}} \mathrm{HPG}$ Alexa Fluor ${ }^{\text {TM }} 594$ Protein Synthesis Assay“(Invitrogen) according to manufactors instructions. Experiments were performed with 6 biological replicates per cell line.

\section{Declarations}

Ethical Approval and Consent to participate

Not applicable

Consent for publication 
All authors give consent for the publication of the manuscript in Cell \& Bioscience

\section{Availability of data and materials}

The datasets used and analyzed during the current study are available from the corresponding author on reasonable request.

\section{Competing interests}

The authors declare that they have no competing interests.

\section{Funding}

This work has been conducted and performed within the framework of the DFG grants MA 1876/12-1 and MA 1876/13-1.

\section{Authors' contributions}

Cloning, sequencing,aquisition of data, analysis and interpretation of data (e.g., statistical analysis, biostatistics, computational analysis): ALS, EK. Constructing the database, visualization of data, writing and reviewing the original draft, of the manuscript, project administration: RM

\section{Acknowledgements}

We are grateful for the kindness of Prof Akihiko Yokoyama who has provide us with his mini-MLL-AF4 constructs. I am also thankful for the helping hands in cell culture and cloning, Silvia Bracharz and Jenny Reis, and for all the fruitful discussions inside the group.

\section{References}

1. Lin S, Luo RT, Ptasinska A, Kerry J, Assi SA, Wunderlich M, et al. Instructive Role of MLL-Fusion Proteins Revealed by a Model of $\mathrm{t}(4 ; 11)$ Pro-B Acute Lymphoblastic Leukemia. Cancer Cell. 2016;30:737-49.

2. Okuda $\mathrm{H}$, Kanai A, Ito $\mathrm{S}$, Matsui $\mathrm{H}$, Yokoyama A. AF4 uses the SL1 components of RNAP1 machinery to initiate MLL fusion- and AEP-dependent transcription. Nat Commun. 2015;6:8869.

3. Meyer C, Burmeister T, Gröger D, Tsaur G, Fechina L, Renneville A, et al. The MLL recombinome of acute leukemias in 2017. Leukemia. 2018;32:273-284.

4. Yokoyama A, Wang Z, Wysocka J, Sanyal M, Aufiero DJ, Kitabayashi I, et al. Leukemia protooncoprotein MLL forms a SET1-like histone methyltransferase complex with menin to regulate Hox gene expression. Mol Cell Biol. 2004;24:5639-49.

5. Milne TA, Dou Y, Martin ME, Brock HW, Roeder RG, Hess JL. MLL associates specifically with a subset of transcriptionally active target genes. Proc Natl Acad Sci USA. 2005;102:14765-70. 
6. Dou Y, Milne TA, Ruthenburg AJ, Lee S, Lee JW, Verdine GL, et al. Regulation of MLL1 H3K4 methyltransferase activity by its core components. Nat Struct Mol Biol. 2006;13:713-19.

7. Bitoun E, Oliver PL, Davies KE. The mixed-lineage leukemia fusion partner AF4 stimulates RNA polymerase II transcriptional elongation and mediates coordinated chromatin remodeling. Hum Mol Genet. 2007;16:92-106.

8. Benedikt A, Baltruschat S, Scholz B, Bursen A, Arrey TN, Meyer B, et al. The leukemogenic AF4-MLL fusion protein causes P-TEFb kinase activation and altered epigenetic signatures. Leukemia. 2011;25:135-44.

9. Luo Z, Lin C, Shilatifard A. The super elongation complex (SEC) family in transcriptional control. Nat Rev Mol Cell Biol. 2012;13:543-7.

10. Mück F, Bracharz S, Marschalek R. DDX6 transfers P-TEFb kinase to the AF4/AF4N (AFF1) super elongation complex. Am J Blood Res. 2016;6:28-45.

11. Benedikt A, Baltruschat S, Scholz B, Bursen A, Arrey TN, Meyer B, et al. The leukemogenic AF4-MLL fusion protein causes P-TEFb kinase activation and altered epigenetic signatures. Leukemia. 2011;25:135-44.

12. Cho S, Schroeder S, Ott M. CYCLINg through transcription: posttranslational modifications of P-TEFb regulate transcription elongation. Cell Cycle. 2010;9:1697-705.

13. Marschalek R. The reciprocal world of MLL fusions: A personal view. Biochim Biophys Acta Gene Regul Mech. 12020;863:194547.

14. Bursen A, Schwabe K, Rüster B, Henschler R, Ruthardt M, Dingermann T, et al. The AF4-MLL fusion protein is capable of inducing ALL in mice without requirement of MLL-AF4. Blood. 2010;115:3570-9.

15. Wilkinson AC, Ballabio E, Geng H, North P, Tapia M, Kerry J, et al. RUNX1 is a key target in $t(4 ; 11)$ leukemias that contributes to gene activation through an AF4-MLL complex interaction. Cell Rep. 2013;3:116-27.

16. Marschalek R. Another piece of the puzzle added to understand $t(4 ; 11)$ leukemia better. Haematologica. 2019;104(6):1098-1100.

17. Secker KA, Bruns L, Keppeler H, Jeong J, Hentrich T, Schulze-Hentrich JM, et al. Only Hematopoietic Stem and Progenitor Cells from Cord Blood Are Susceptible to Malignant Transformation by MLLAF4 Translocations. Cancers (Basel). 2020;12:1487.

18. Okuda H, Takahashi S, Takaori-Kondo A, Yokoyama A. TBP loading by AF4 through SL1 is the major rate-limiting step in MLL fusion-dependent transcription. Cell Cycle. 2016;15:2712-22.

19. Learned RM, Cordes S, Tjian R. Purification and characterization of a transcription factor that confers promoter specificity to human RNA polymerase I. Mol Cell Biol. 1985;5:1358-69.

20. Hempel WM, Cavanaugh AH, Hannan RD, Taylor L, Rothblum LI. The species-specific RNA polymerase I transcription factor SL-1 binds to upstream binding factor. Mol Cell Biol. 1996;16:55763. 
21. Tuan JC, Zhai W, Comai L. Recruitment of TATA-binding protein-TAFI complex SL1 to the human ribosomal DNA promoter is mediated by the carboxy-terminal activation domain of upstream binding factor (UBF) and is regulated by UBF phosphorylation. Mol Cell Biol. 1999;19:2872-9.

22. Hochheimer A, Tjian R. Diversified transcription initiation complexes expand promoter selectivity and tissue-specific gene expression. Genes Dev. 2003;17:1309-20.

23. Friedrich JK, Panov KI, Cabart P, Russell J, Zomerdijk JC. TBP-TAF complex SL1 directs RNA polymerase I pre-initiation complex formation and stabilizes upstream binding factor at the rDNA promoter. J Biol Chem. 2005;280:29551-8.

24. Bross K, Krone W. On the number of ribosomal RNA genes in man. Humangenetik. 1972;14:137-41.

25. Grummt I, Längst G. Epigenetic control of RNA polymerase I transcription in mammalian cells. Biochim Biophys Acta. 2013;1829:393-404.

26. Dannheisig DP, Bächle J, Tasic J, Keil M, Pfister AS. The Wnt/B-Catenin Pathway is Activated as a Novel Nucleolar Stress Response. J Mol Biol. 2021;433:166719.

27. Yokoyama A. RNA Polymerase II-Dependent Transcription Initiated by Selectivity Factor 1: A Central Mechanism Used by MLL Fusion Proteins in Leukemic Transformation. Front Genet. 2019;9:722.

28. Kowarz E, Löscher D, Marschalek R. Optimized Sleeping Beauty transposons rapidly generate stable transgenic cell lines. Biotechnol J. 2015;10:647-653.

29. Caslini C, Serna A, Rossi V, Introna M, Biondi A. Modulation of cell cycle by graded expression of MLL-AF4 fusion oncoprotein. Leukemia. 2004;18:1064-71.

30. Wang Y, Krivtsov AV, Sinha AU, North TE, Goessling W, Feng Z, Zon LI, Armstrong SA. The Wnt/betacatenin pathway is required for the development of leukemia stem cells in AML. Science. 2010;327:1650-3.

31. 31.Fung TK, Gandillet A, So CW. Selective treatment of mixed-lineage leukemia leukemic stem cells through targeting glycogen synthase kinase 3 and the canonical Wnt/ $\beta$-catenin pathway. Curr Opin Hematol. 2012;19:280-6.

32. Walf-Vorderwülbecke V, de Boer J, Horton SJ, van Amerongen R, Proost N, Berns A, Williams O. Frat2 mediates the oncogenic activation of Rac by MLL fusions. Blood. 2012;120:4819-28.

33. Abraham KJ, Khosraviani N, Chan JNY, Gorthi A, Samman A, et al. Nucleolar RNA polymerase II drives ribosome biogenesis. Nature. 2020;585:298-302.

\section{Figures}


A
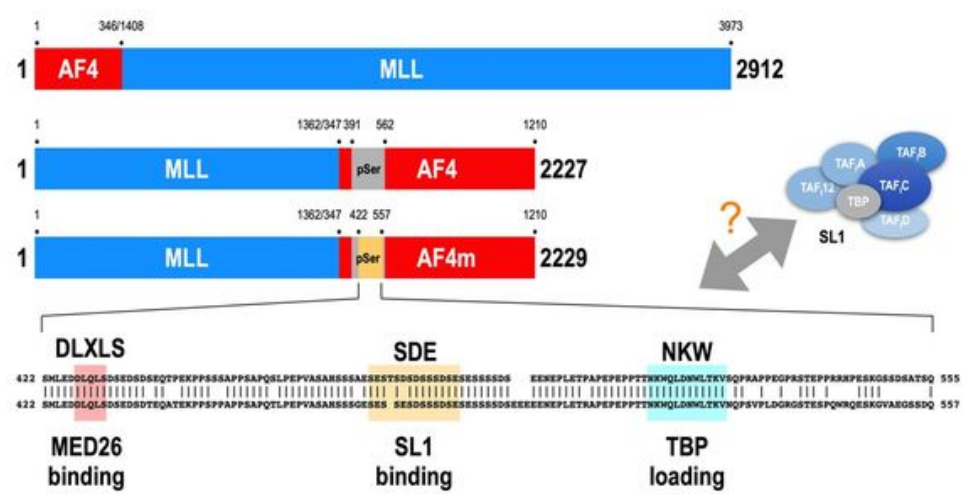

B

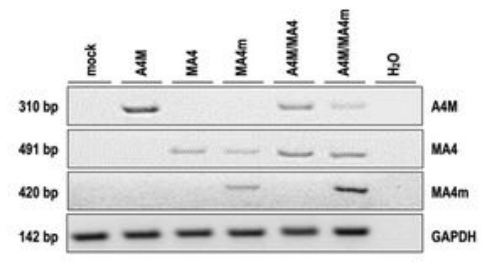

D

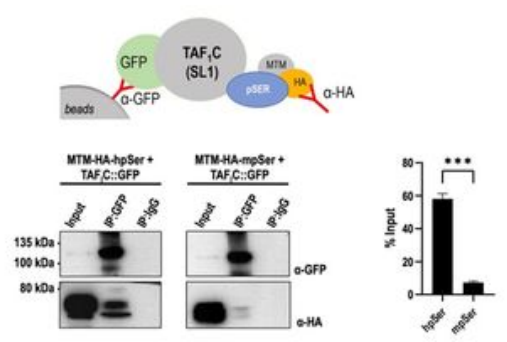

$\mathbf{F}$
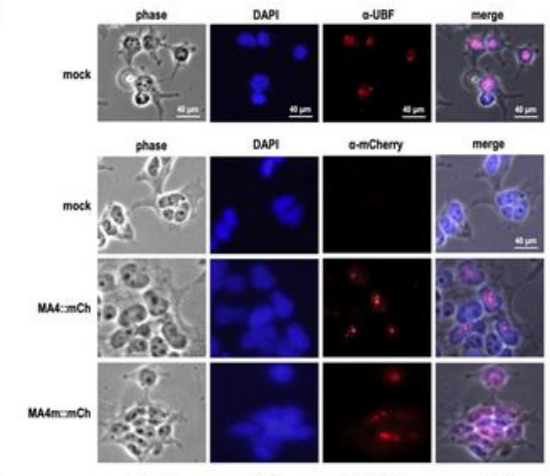

G

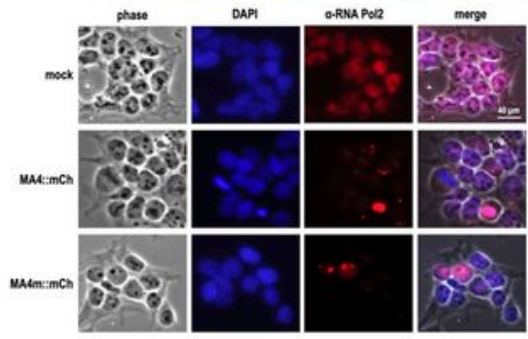

C

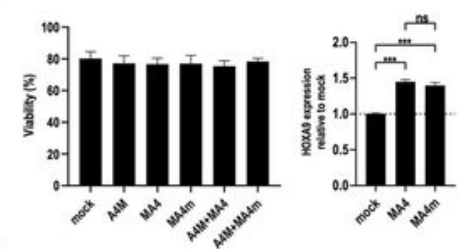

E
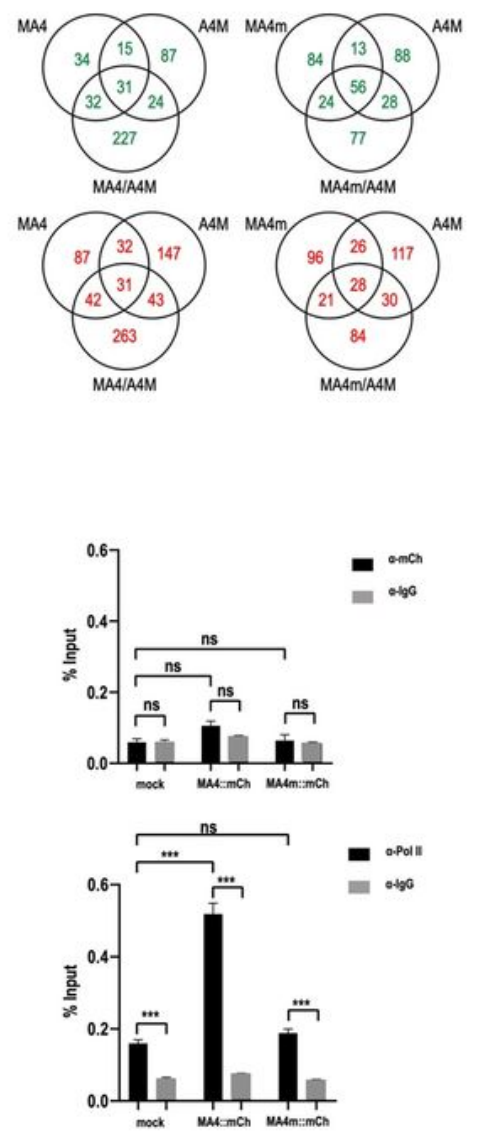

\section{Figure 1}

Functional studies using the expression constructs MA4, MA4m and A4M A.Design of A4M, MA4 and MA4m transgenes. Amino acids coordinates of fused portions and the pSer domain are indicated. The exchanged pSer domain contained the binding sites for the Mediator complex, SL1 and TBP. B. RT-PCR analyses of all inducible transgenes. These experiments validated the correct expression of all stably transfected transgenes. Sizes of each amplimer are given to the left. A GAPDH primer set was used to 
validate that equal amounts of cellular RNA were used in all experiments. C. Cell viability and target gene validation. Cell viability was tested in independent experiments $(n=3)$. A single downstream target genes, HOXA9, was tested to validate the functionality of both the MA4 and MA4m fusion gene constructs. D.Coimmunprecipitation validated the binding of SL1 to the human pSer domain, while the murinized version binds to a much lesser extent. E. MACE-Seq experiment revealed the synergism between MA4 and A4M. MA4m has lost this ability. F. Immunohistology experiments performed with mCherry tagged MA4 and MA4m constructs. Only MA4 was able to relocalize RNA Pol II from the nucleus to the nucleolus.

A

B
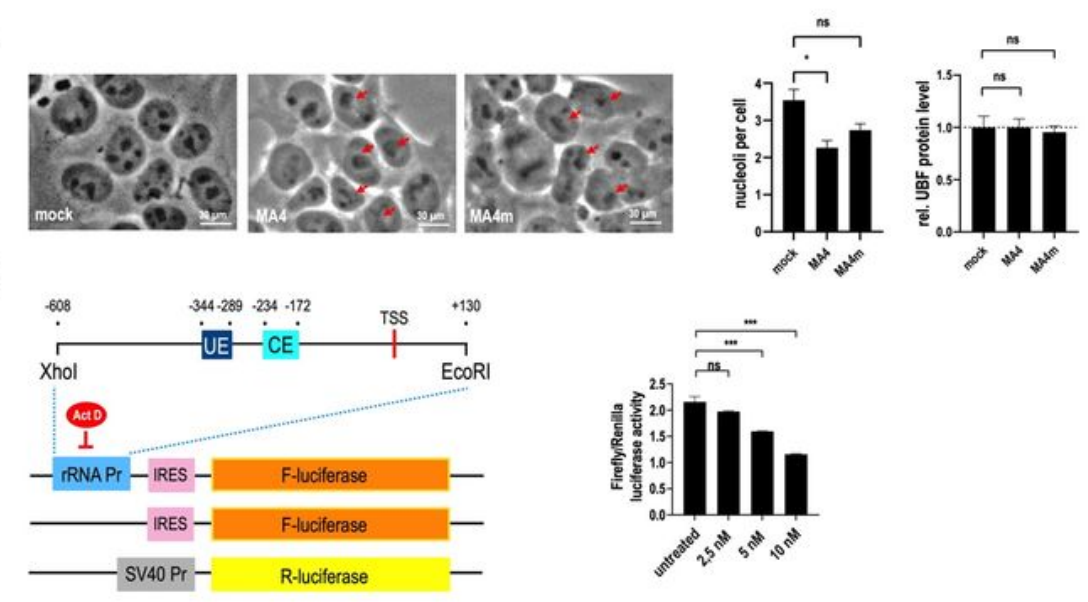

C

D
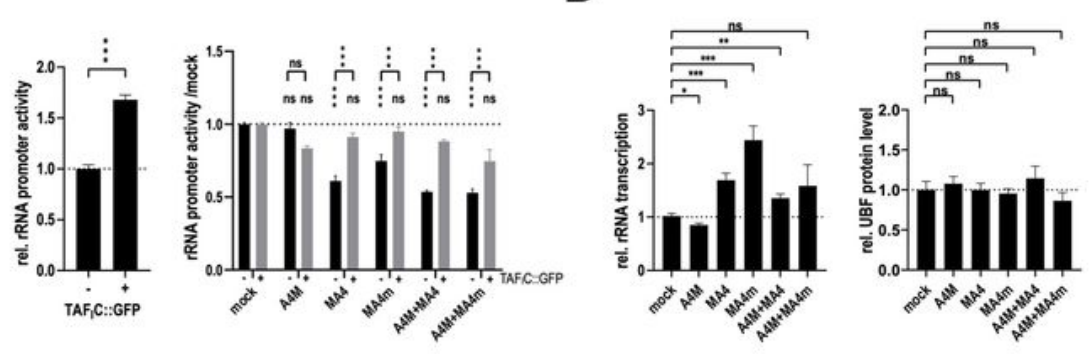

E

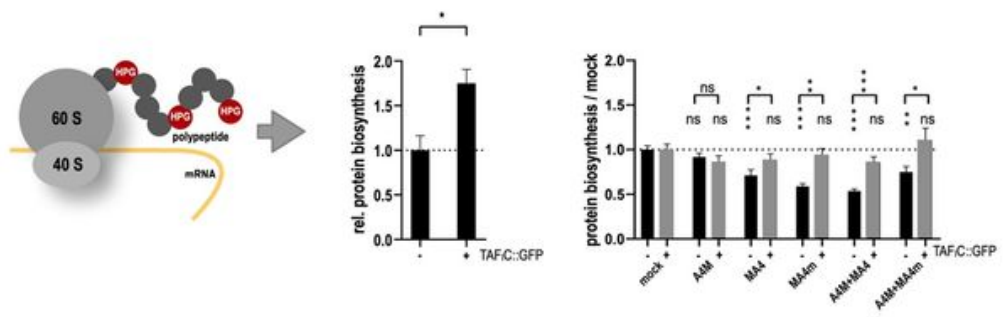

F
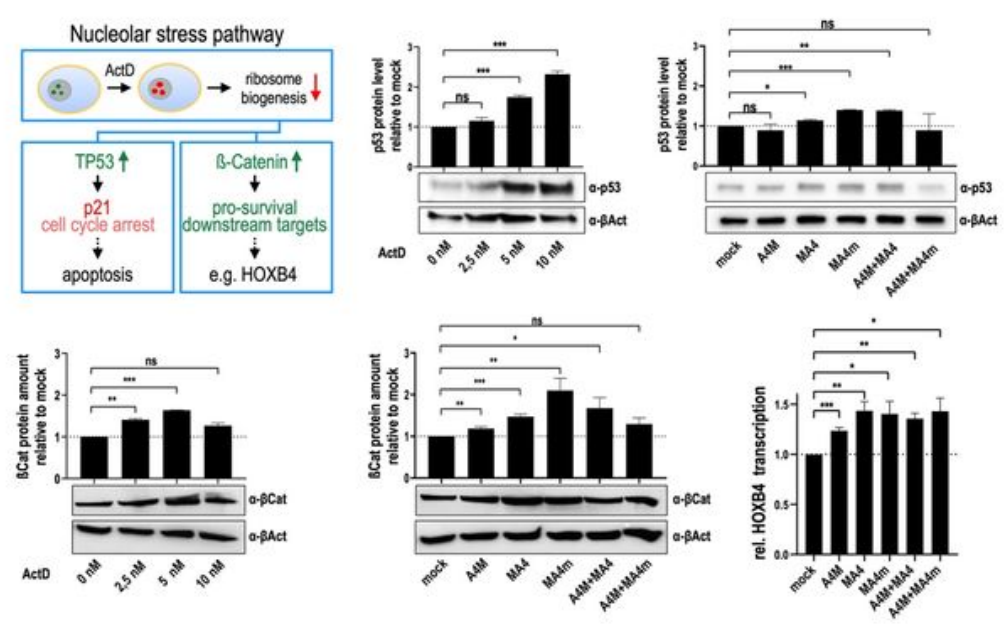


\section{Figure 2}

Nucleolus, rRNA synthesis and protein biosynthesis A.Morphology of nucleoli after transfection with MA4 and MAF4m. The morphology and number of nucleoli is grossly changed in the presence of both fusion proteins, MA4 and MA4m, respectively. B.Design of the RNA Pol I Luciferase reporter gene. A single rRNA promoter has been used to set up a RNA Pol I dependent Luciferase reporter system that is responsive to Actinomycin D. C. Reporter gene assays to monitor rRNA promoter activity. The TAFIC::GFP fusion was able to enhance rRNA promoter activity. All fusion gene expression cell lines showed a reduction of rRNA promoter activity. D. Q-PCR experiments of 45S rRNA precursors. Apart from A4M-single transfected cells, all others displayed increasing amount of the 45S precursor RNA. The amount of UBF protein was quantified and remained stable in all cell lines. E. Protein biosynthesis measured by the Click iT protein synthesis assay. Apart from A4M-single transfected cells, while all others displayed a decreased protein biosynthesis rate. F. Investigating the nucleolar stress pathway. Either ActD or the presence of $t(4 ; 11)$ fusion proteins caused the steady-state upregulation of p53 and B-Catenin. A downstream target of the WNT/ß-Catenin signaling pathway, HOXB4, was significantly upregulated by all tested fusion proteins.

\section{Supplementary Files}

This is a list of supplementary files associated with this preprint. Click to download.

- A4Mgenesignature.xlsx

- A4Mvolcano.xlsx

- HeatmapDataset1.xlsx

- HeatmapDataset2.xlsx

- MA4genesignature.xlsx

- MA4volcano.xlsx

- MA4A4Mgenesignature.xlsx

- MA4A4Mvolcano.xIsx

- MA4mgenesignature.xlsx

- MA4mvolcano.xlsx

- MA4mA4Mgenesignature.xlsx

- MA4mA4Mvolcano.xlsx

- SupplementalDatafile.pdf 